MPI-PhT/95-57

CCNY-HEP-95/5

hep-ph/9604341

July 1995

\title{
Bimodal Coherence in Dense Self-Interacting Neutrino Gases
}

\author{
Stuart Samuel* \\ Max-Planck-Institut für Physik \\ Werner-Heisenberg-Institut \\ Föhringer Ring 6 \\ 80805 Munich, Germany
}

\begin{abstract}
Analytical solutions are obtained to the nonlinear equations describing neutrino oscillations when explicit neutrino-antineutrino asymmetries are present. Such a system occurs in the early Universe if neutrinos have a non-zero chemical potential. Solutions to the equations lead to a new type of coherent behavior governed by two modes. These bimodal solutions provide new insights into dense neutrino gases and into neutrino oscillations in the early Universe, thereby allowing one to surmise the flavor behavior of neutrinos with a non-zero chemical potential.
\end{abstract}

*Permanent address and address after August, 1995:

Physics Department, City College of New York, New York, NY 10031, USA.

E-mail: samuel@scisun.sci.ccny.cuny.edu 


\section{Introduction}

In this work we undertake a theoretical study of flavor oscillations in dense neutrino gases. Such gases appear in physically interesting systems. An example occurs during the early Universe when neutrinos are self-interacting and fill space densely [1, 2]. Likewise, during the final collapse of a supernova, neutrinos are emitted copiously [2, [3]. If neutrinos have masses and mix then oscillations among neutrino flavors can effect the physics of these systems.

The treatment of neutrino oscillations in a dense gas is not so straightforward. The flavor behavior of a particular neutrino depends on the flavor content of background neutrinos. However, the background neutrinos also oscillate. To know the flavor content of background neutrinos, it is necessary to know the oscillations of all individual neutrinos. Nonetheless, a Hartree-Fock-like self-consistent formalism has appeared which can handle the system [4]. The behavior of the gas depends on certain statistical properties such as the energy distribution, the nature of density perturbations and the initial production of neutrinos. For the case in which only an energy distribution is involved, the formalism has been further developed for the neutrino-antineutrino gas in refs. [5, 6, [7]. The physical effects of neutrino oscillations on supernovae is treated in ref. [8].

Since the system is self-interacting and nonlinear, one usually has to resort to numerical methods to determined the physics and flavor behavior. This approach was used in refs. 6, 0, 9, 10, 11] to analyze neutrino oscillations in the early Universe for the case in which neutrinos have a chemical potential $\mu_{\nu}$ which is zero. In a gas for which $\mu_{\nu}=0$, the total number of antineutrinos is equal to the total number of neutrinos. It is unknown whether $\mu_{\nu}=0$ in the early Universe. Indeed, the excess of electrons over positrons implies that the chemical potential for charged leptons is nonzero. Because this excess is tiny, being related to the baryon asymmetry of the Universe, the chemical potential for electrons is quite small. Nevertheless, because neutrinos are so dense at one second after the Big Bang, when the temperature of the Universe is about an $\mathrm{MeV}$, even a tiny $\mu_{\nu}$ can have an effect. One purpose of the current work is to surmise the flavor behavior of neutrino oscillations in the early 
Universe when $\mu_{\nu} \neq 0$. Various aspects of neutrino oscillations in the early Universe are also addressed in refs. [12-20].

Thus one is led to consider the system when the total number of antineutrinos is not necessarily equal to the total number of neutrinos. We assume the gas is homogeneous and isotropic. Under this assumption, the averaged spatial neutrino currents are zero and one needs only to consider neutrino densities. We also assume that the energy distribution is the most important statistical property of the gas. For simplicity, two flavors, electron and muon, are treated. In many regions of parameter space, the three-flavor case is accurately approximated by the two-flavor case. Finally, we consider situations in which hard scattering processes are much smaller than forwardscattering phase effects so that hard scattering can be and is ignored. This occurs for times greater than one second after the Big Bang and outside the neutrino sphere of a supernova. In these situations, $G_{F} E^{2} \ll 1$, where $G_{F} \simeq 1.17 \times 10^{-11} \mathrm{MeV}^{-2}$ is the Fermi coupling constant and $E$ is the energy of a typical neutrino. The analytic results as well as the graphs displayed in the figures hold only for the case in which non-forward scattering can be neglected.

Several parameters describe the system. Two important ones are $\Delta$, which is the mass squared difference between the two mass-eigenstate neutrinos, and $\theta$, which is the vacuum mixing angle:

$$
\Delta=\left(m_{2}^{2}-m_{1}^{2}\right)
$$

and

$$
\nu_{1}=\nu_{e L} \cos \theta-\nu_{\mu L} \sin \theta \quad, \quad \nu_{2}=\nu_{e L} \sin \theta+\nu_{\mu L} \cos \theta \quad .
$$

Here, $\nu_{1}$ and $\nu_{2}$ are the mass-eigenstate neutrinos, which have masses $m_{1}$ and $m_{2}$, and $\nu_{e L}$ and $\nu_{\mu L}$ are the left-handed electron and muon neutrinos, which are the states entering in the currents of the weak interactions.

Let $N_{\nu}$ and $N_{\bar{\nu}}$ be respectively the number of neutrinos and the number of antineutrinos in the gas. We use labels $j$ and $k$ to enumerate respectively the neutrinos and the antineutrinos. Thus the index $j$ runs from 1 to $N_{\nu}$ and the index $k$ runs from 1 to $N_{\bar{\nu}}$. The energy of the $j$ th neutrino and the $k$ th antineutrino is denoted by $E^{j}$ and $\bar{E}^{k}$. The system is put in a box of volume $\mathcal{V}$. The neutrino and antineutrino 
densities $n_{\nu}$ and $n_{\bar{\nu}}$ are given by $n_{\nu}=N_{\nu} / \mathcal{V}$ and $n_{\bar{\nu}}=N_{\bar{\nu}} / \mathcal{V}$.

The wave function for $j$ th neutrino is governed by a two-component vector $\nu^{j}$ in flavor space, i.e.,

$$
\nu^{j}=\left(\begin{array}{c}
\nu_{e}^{j} \\
\nu_{\mu}^{j}
\end{array}\right)
$$

where $\nu_{e}^{j *} \nu_{e}^{j}$ (respectively, $\nu_{\mu}^{j *} \nu_{\mu}^{j}$ ) is the probability that the $j$ th neutrino is an electron neutrino (respectively, muon neutrino). Likewise, for the $k$ th antineutrino, the flavor wave function is

$$
\bar{\nu}^{k}=\left(\begin{array}{c}
\bar{\nu}_{e}^{k} \\
\bar{\nu}_{\mu}^{k}
\end{array}\right)
$$

Since, under the assumption of no hard scattering, neutrinos and antineutrinos are not created or destroyed but merely change flavor, conservation of probability implies that

$$
\nu_{e}^{j *} \nu_{e}^{j}+\nu_{\mu}^{j *} \nu_{\mu}^{j}=1, \quad \bar{\nu}_{e}^{k *} \bar{\nu}_{e}^{k}+\bar{\nu}_{\mu}^{k *} \bar{\nu}_{\mu}^{k}=1
$$

The equations governing neutrino oscillations are easier to understand intuitively and visually by using vectors $\vec{v}^{j}$ and $\vec{w}^{k}$ associated with Eqs. (1.3) and (1.4) given by 13

$$
\begin{aligned}
\vec{v}^{j} & \equiv\left(\nu_{e}^{j *} \nu_{e}^{j}-\nu_{\mu}^{j *} \nu_{\mu}^{j}, 2 \operatorname{Re}\left(\nu_{e}^{j *} \nu_{\mu}^{j}\right), 2 \operatorname{Im}\left(\nu_{e}^{j *} \nu_{\mu}^{j}\right)\right) \\
\vec{w}^{k} & \equiv\left(\bar{\nu}_{e}^{k *} \bar{\nu}_{e}^{k}-\bar{\nu}_{\mu}^{k *} \bar{\nu}_{\mu}^{k}, 2 \operatorname{Re}\left(\bar{\nu}_{e}^{k *} \bar{\nu}_{\mu}^{k}\right), 2 \operatorname{Im}\left(\bar{\nu}_{e}^{k *} \bar{\nu}_{\mu}^{k}\right)\right)
\end{aligned}
$$

where Re and Im denote the real and imaginary parts of a complex number.

The equations governing the flavor behavior of a gas of self-interacting neutrinos and antineutrinos are [4, 5, 6, 20]

$$
\frac{d \vec{v}^{j}}{d t}=\vec{v}^{j} \times \vec{B}_{v}^{j}, \quad \frac{d \vec{w}^{k}}{d t}=\vec{w}^{k} \times \vec{B}_{w}^{k},
$$

where $\vec{B}_{v}^{j}$ and $\vec{B}_{w}^{k}$ are given by

$$
\vec{B}_{v}^{j}=\frac{\vec{\Delta}}{2 E^{j}}-\vec{V}_{\nu \nu}, \quad \vec{B}_{w}^{k}=\frac{\vec{\Delta}}{2 \bar{E}^{k}}+\vec{V}_{\nu \nu}^{*}
$$

with

$$
\vec{\Delta} \equiv \Delta(\cos 2 \theta,-\sin 2 \theta, 0)
$$

Here,

$$
\vec{V}_{\nu \nu}=\frac{\sqrt{2} G_{F}}{\mathcal{V}}\left(\langle\vec{v}\rangle-\left\langle\vec{w}^{*}\right\rangle\right)
$$


implements the interactions of neutrinos and antineutrinos among themselves. An asterisk on a vector indicates a change in sign of the third component so that $\vec{w}^{*}=$ $\left(w_{1}, w_{2},-w_{3}\right)$. In Eq. (1.10),

$$
\langle\vec{v}\rangle=\sum_{j} \vec{v}^{j}, \quad\langle\vec{w}\rangle=\sum_{k} \vec{w}^{k}
$$

The neutrino-neutrino term $\vec{V}_{\nu \nu}$ makes the system of equations in Eq. (1.7) nonlinear.

The equations in Eq. (1.7) resemble the motion of charged particles in "magnetic fields" $\vec{B}_{v}^{j}$ and $\vec{B}_{w}^{k}$.

The conservation of individual neutrinos in Eq. (1.5) corresponds to

$$
\vec{v}^{j} \cdot \vec{v}^{j}(t)=1, \quad \vec{w}^{k} \cdot \vec{w}^{k}(t)=1
$$

The total number of neutrinos $N_{\nu}$ and antineutrinos $N_{\bar{\nu}}$ is thus $N_{\nu}=\sum_{j}\left|\vec{v}^{j}\right|$ and $N_{\bar{\nu}}=\sum_{k}\left|\vec{w}^{k}\right|$.

If, at time $t=0$, the $j$ th neutrino and $k$ th antineutrino begin as an electron neutrino and an electron antineutrino then

$$
\vec{v}^{j}(0)=(1,0,0), \quad \vec{w}^{k}(0)=(1,0,0)
$$

If they begin as a muon neutrino and muon antineutrino then $\vec{v}^{j}(0)=(-1,0,0)$ and $\vec{w}^{k}(0)=(-1,0,0)$. If there are both electron and muon neutrinos initially, then only the excess is relevant for neutrino oscillations. Thus if the there are more electron neutrinos than muon neutrinos at $t=0$ then Eq. (1.13) is general. In the current work, the initial conditions in Eq. (1.13) are referred to as initial flavor eigenstates.

\section{Reduction of the Problem}

The ratio of the strength of the vacuum term to the maximum possible neutrinointeraction term for the $j$ th neutrino and $k$ th antineutrino is given by the parameters $\kappa^{j}$ and $\bar{\kappa}^{k}$, where

$$
\kappa^{j}=\frac{\Delta}{\sqrt{2} G_{F} E^{j}\left(n_{\nu}+n_{\bar{\nu}}\right)}, \quad \bar{\kappa}^{k}=\frac{\Delta}{\sqrt{2} G_{F} \bar{E}^{k}\left(n_{\nu}+n_{\bar{\nu}}\right)} .
$$

When $\kappa^{j} \ll 1$ and $\bar{\kappa}^{k} \ll 1$, the vacuum term is much smaller than the nonlinear neutrino term. This corresponds to the case when the neutrino gas is dense. Under 
these circumstances, ref. [7] found that alignment holds. Alignment has been observed numerically [7] and is understood theoretically [21]. When alignment holds,

$$
\vec{v}^{j}(t) \approx \frac{\langle\vec{v}\rangle}{N_{\nu}} \equiv \vec{r}_{v}(t), \quad \vec{w}^{k}(t) \approx \frac{\langle\vec{w}\rangle}{N_{\bar{\nu}}} \equiv \vec{r}_{w}(t)
$$

are good approximations. Throughout the rest of this work, the dense neutrino case is treated, so that Eq. (2.2) hold quite accurately.

When Eq. (2.2) is an exact equality, the system of $3 N_{\nu}+3 N_{\bar{\nu}}$ differential equations in Eq. (1.7) reduce to six equations. One sums over $j$ and $k$ in Eq. (1.7) to obtain 21]

$$
\frac{d \vec{r}_{v}}{d t}=\vec{r}_{v} \times\left(\frac{\vec{\Delta}}{2 E_{0}}-\vec{V}_{\nu \nu}\right), \quad \frac{d \vec{r}_{w}}{d t}=\vec{r}_{w} \times\left(\frac{\vec{\Delta}}{2 \bar{E}_{0}}+\vec{V}_{\nu \nu}^{*}\right)
$$

where

$$
\vec{V}_{\nu \nu}=\sqrt{2} G_{F}\left(n_{\nu} \vec{r}_{v}-n_{\bar{\nu}} \vec{r}_{w}^{*}\right)
$$

and $1 / E_{0}$ and $1 / E_{0}$ are the average inverse neutrino and antineutrino energies given by

$$
\frac{1}{E_{0}} \equiv \frac{1}{N_{\nu}} \sum_{j} \frac{1}{E^{j}}, \quad \frac{1}{\bar{E}_{0}} \equiv \frac{1}{N_{\bar{\nu}}} \sum_{k} \frac{1}{\bar{E}^{k}} .
$$

Initial flavor eigenstates correspond to

$$
\vec{r}_{v}(0)=(1,0,0), \quad \vec{r}_{w}(0)=(1,0,0)
$$

\section{The Solution}

It is convenient to change variables. First, we use $\tau$ in lieu of $t$, where

$$
\tau \equiv \frac{1}{2} \frac{\Delta}{E_{0}} \sqrt{\frac{1}{\kappa_{0}}} t
$$

with

$$
\kappa_{0} \equiv \frac{\Delta}{2 \sqrt{2} G_{F} E_{0} n_{\nu}}
$$

Second, we use a mass eigenstate basis. This involves a rotation of vectors by an angle of $2 \theta$. We denote the new vectors for neutrinos by $\vec{y}$ and for antineutrinos by $\vec{z}$. They are given by

$$
y_{1}(\tau) \equiv r_{v 1}(t) \cos 2 \theta-r_{v 2}(t) \sin 2 \theta
$$




$$
\begin{gathered}
y_{2}(\tau) \equiv r_{v 1}(t) \sin 2 \theta+r_{v 2}(t) \cos 2 \theta \\
y_{3}(\tau) \equiv r_{v 3}(t)
\end{gathered}
$$

and

$$
\begin{gathered}
z_{1}(\tau) \equiv \frac{n_{\nu}}{n_{\bar{\nu}}}\left(r_{w 1}(t) \cos 2 \theta-r_{w 2}(t) \sin 2 \theta\right) \\
z_{2}(\tau) \equiv \frac{n_{\nu}}{n_{\bar{\nu}}}\left(r_{w 1}(t) \sin 2 \theta+r_{w 2}(t) \cos 2 \theta\right) \\
z_{3}(\tau) \equiv-\frac{n_{\nu}}{n_{\bar{\nu}}} r_{w 3}(t) .
\end{gathered}
$$

For antineutrinos, a factor of $n_{\nu} / n_{\bar{\nu}}$ is included for convenience, and the sign of the third component is flipped, which corresponds to using the "*” vector. Equation (2.3) becomes

$$
\frac{d \vec{y}}{d \tau}=\vec{y} \times\left(\gamma \hat{e}_{1}-\frac{1}{\gamma}(\vec{y}-\vec{z})\right), \quad \frac{d \vec{z}}{d \tau}=-\vec{z} \times\left(\bar{\gamma} \hat{e}_{1}+\frac{1}{\gamma}(\vec{y}-\vec{z})\right)
$$

where

$$
\gamma \equiv \sqrt{\kappa_{0}}, \quad \bar{\gamma} \equiv \frac{E_{0}}{\bar{E}_{0}} \gamma
$$

Initial flavor eigenstates correspond to

$$
\vec{y}(0)=(\cos 2 \theta, \sin 2 \theta, 0), \quad \vec{z}(0)=\frac{n_{\nu}}{n_{\bar{\nu}}}(\cos 2 \theta, \sin 2 \theta, 0)
$$

Equation (3.5) is solved for arbitrary initial conditions in Appendix A. The solution for $y_{1}$ and $w_{1}$ is in terms of Jacobi elliptic functions. Thus the motion of the first components of $\vec{y}$ and $\vec{w}$ is period in $\tau$ with a period which we denote by $\mathcal{T}_{f}$. See Eqs. (A.18) and (A.20). The solutions for the second and third components are

$$
\begin{gathered}
y_{2}(\tau)=\sqrt{1-y_{1}^{2}(\tau)} \cos \varphi(\tau), \quad y_{3}(\tau)=\sqrt{1-y_{1}^{2}(\tau)} \sin \varphi(\tau) \\
z_{2}(\tau)=\sqrt{\left(\frac{n_{\nu}}{n_{\bar{\nu}}}\right)^{2}-\left(k_{1}-y_{1}(\tau)\right)^{2}} \cos \bar{\varphi}(\tau) \\
z_{3}(\tau)=\sqrt{\left(\frac{n_{\nu}}{n_{\bar{\nu}}}\right)^{2}-\left(k_{1}-y_{1}(\tau)\right)^{2}} \sin \bar{\varphi}(\tau)
\end{gathered}
$$

where $k_{1}=y_{1}(0)-z_{1}(0)$ and where $\varphi$ and $\bar{\varphi}$ are complicated functions of $y_{1}$. Because $y_{1}$ is periodic, $\cos \varphi(\tau)$ and $\sin \varphi(\tau)$ are periodic with a period $\mathcal{T}_{s}$. Likewise, $\cos \bar{\varphi}(\tau)$ 
and $\sin \bar{\varphi}(\tau)$ are periodic with a period $\overline{\mathcal{T}}_{s}$. See Eq. A.25). The periods in $t$ are related to those in $\tau$ by the factor $2 E_{0} \sqrt{\kappa_{0}} / \Delta$ according to Eq. (3.1).

Hence, the motion of the second and third components is bimodal. They involve two periodic functions each with a different period. In terms of the original variables $\vec{r}_{v}$ and $\vec{r}_{w}$, all components are bimodal because $r_{v 1}$ and $r_{w 1}$ involve $y_{2}$ and $w_{2}$ when Eqs. (3.3) and (3.4) are inverted. In general, the periods are not compatible and the curves for $r_{v 1}, r_{v 2}, r_{v 3}, r_{w 1}, r_{w 2}$ and $r_{w 3}$ appear irregular. When the gas has only neutrinos and no antineutrinos, the slow mode goes away and the behavior is purely periodic. This was the case observed in ref. [4]. Likewise, in the CP-symmetric case, in which $N_{\nu}=N_{\bar{\nu}}$ and $E_{0}=\bar{E}_{0}$, the periods $\mathcal{T}_{s}$ and $\overline{\mathcal{T}}_{s}$ go to infinity and the motion also becomes periodic with period $\mathcal{T}_{f}$. This is the case treated in ref. [21].

\section{Explicit Examples}

The analytic solution in Appendix A is quite complicated. To obtain insight, it is useful to consider specific cases. Below, the values for $\sin ^{2} 2 \theta, \Delta, n_{\nu}, n_{\bar{\nu}}$, etc., are not chosen to correspond to those in physical systems, such as in the early Universe, but to best illustrate different behaviors.

It is convenient to use $\tau$ in Eq. (3.1) since then the system depends on only four parameters, namely, $\sin 2 \theta, \gamma, \bar{\gamma}$ and $n_{\nu} / n_{\bar{\nu}}$. Although solutions also depend on the initial values of $\vec{y}(0)$ and $\vec{z}(0)$, in this section, we avoid this dependence by using the initial flavor eigenstates in Eq. (3.7). We select $\sin 2 \theta=0.8$. The large value for the vacuum mixing angle amplifies effects.

Figure 1 shows the three components of $\vec{y}(\tau)$ for the case $n_{\nu} / n_{\bar{\nu}}=0.9, \gamma=0.1$ and $\bar{\gamma}=0.12$. Since the solution for $y_{1}(\tau)$ is always in terms of a Jacobi elliptic function, the graph of $y_{1}(\tau)$ is periodic. This is evident in Figure 1a. Since two periods are involved in $y_{2}(\tau)$ and $y_{3}(\tau)$, the corresponding curves are bimodal and irregular, as Figures $1 \mathrm{~b}$ and $1 \mathrm{c}$ show. The plots for $z_{1}(\tau), z_{2}(\tau)$ and $z_{3}(\tau)$ are qualitatively similar to $y_{1}(\tau), y_{2}(\tau)$ and $y_{3}(\tau)$. In other words, $z_{1}(\tau)$ is periodic, and $z_{2}(\tau)$ and $z_{3}(\tau)$ are bimodal and irregular. To save space, we do not display them. Additional insight is gained by examining the orbit for neutrinos, i.e., the trajectory $\left(y_{1}(\tau), y_{2}(\tau), y_{3}(\tau)\right)$ 
in three-space. Figure 2a displays the neutrino orbit. The motion is as though one were moving back and forth over a cup-shaped surface in which the cup is also slowly rotating. These two motions crudely represent the two modes: The back-and-forth mode has a period of $\mathcal{T}_{f}$. The other mode corresponds to the rotation of the cup, is governed by the period $\mathcal{T}_{s}$, and is controlled by $\varphi(\tau)$. The antineutrino orbit, given by $\vec{z}(\tau)$, is similar to $\vec{y}(\tau)$ except that "little loops" are made near the "edge" of the cup. See Figure 2b. In the flavor eigenstate basis, all components are bimodal and irregular. Figure 3 displays $r_{v 1}(\tau) \equiv \cos 2 \theta y_{1}(\tau)+\sin 2 \theta y_{2}(\tau)$. Qualitatively $r_{w 1}(\tau)$ is similar to $r_{v 1}(\tau)$. The case in this paragraph is near the CP-symmetric limit for which $n_{\nu} / n_{\bar{\nu}}=1.0$ and $\gamma=\bar{\gamma}$. In that limit, the motion is back-and-forth over the cup but the cup does not rotate [21] so that the motion is a single mode and periodic in all variables.

When $n_{\nu} / n_{\bar{\nu}}$ is reduced, the amplitude for the back-and-forth motion becomes smaller and does not pass over the "top" of the cup. Figure 4a shows the orbit of $\vec{y}(\tau)$ for the case $n_{\nu} / n_{\bar{\nu}}=0.75, \gamma=0.1$ and $\bar{\gamma}=0.12$. The antineutrino orbit is similar to the neutrino orbit and again has little loops. See Figure 4b. In terms of components, $y_{1}(\tau)$ and $z_{1}(\tau)$ have graphs similar to the one in Figure 1a but the amplitude is smaller. The graphs for $y_{2}(\tau), y_{3}(\tau), z_{2}(\tau), z_{3}(\tau)$ look like cosine and sine functions with "small bumps". Figure 5, the plot of $y_{2}$ versus $\tau$, exemplifies this.

When $n_{\nu} / n_{\bar{\nu}}=0$, one obtains the pure neutrino gas with no antineutrinos. The orbit for the neutrinos in this limiting case, which was analytically obtained in ref. [21], is a circle. Figure 6a displays the neutrino orbit for the case $n_{\nu} / n_{\bar{\nu}}=0.4$, $\gamma=0.5$ and $\bar{\gamma}=0.6$. As expected, the orbit is approximately a circle. However, because $n_{\nu} / n_{\bar{\nu}} \neq 0$, there are "wiggles". The orbit looks somewhat like a "lasso". The antineutrino orbit is "flowerlike". See Figure 6b.

It turns out that, in the above three examples, $\overline{\mathcal{T}}_{s}=\mathcal{T}_{s}$. For $\gamma$ and $\bar{\gamma}$ not too large, this equality is general and holds for other values of the parameters $\sin 2 \theta, n_{\nu} / n_{\bar{\nu}}$, etc.. Hence the angular motions, $\varphi(\tau)$ and $\bar{\varphi}(\tau)$, do not get out of phase. As a consequence, the initial CP-asymmetry is not amplified in time. For the discussion in the Section VI, we refer to this phenomenon as $\varphi$-phase locking.

However, when $\gamma$ and $\bar{\gamma}$ become sufficiently large, the equality $\overline{\mathcal{T}_{s}}=\mathcal{T}_{s}$ is suddenly 
violated. For initial flavor conditions, this happens at the point in parameter space when

$$
\frac{n_{\nu}}{n_{\bar{\nu}}}(1-\cos 2 \theta)=y_{+},
$$

where $y_{+}$is given in Eq. (A.17). This is the point at which the denominator factor $\left(n_{\nu} / n_{\bar{\nu}}\right)^{2}-\left(y_{1}-k_{1}\right)^{2}$ in $\frac{d \bar{\varphi}}{d \tau}$ vanishes for the maximum value obtained by $y_{1}$. See Eq. (A.23). For $\gamma$ or $\bar{\gamma}$ above the critical point, $\overline{\mathcal{T}}_{s} \neq \mathcal{T}_{s}$. In this case, even a small initial $\mathrm{CP}$-asymmetry can eventually grow into a large $\mathrm{CP}$-asymmetry at certain times.

As an example of this phenomenon, consider the case with $n_{\nu} / n_{\bar{\nu}}=0.9, \gamma=0.5$ and $\bar{\gamma}=0.5$. One finds $\mathcal{T}_{f} \approx 2.2832, \mathcal{T}_{s} \approx 4.8383, \overline{\mathcal{T}_{s}} \approx 4.3235$. Figures $7 \mathrm{a}$ and $7 \mathrm{~b}$ compare $y_{2}(\tau)$ and $z_{2}(\tau)$. By the time $\tau$ is $10, y_{2}$ and $z_{2}$ are about one-sixth of a period out of phase so that a sizeable CP-asymmetry is built up, even though the initial CP-asymmetry, as measured by the difference of $n_{\nu} / n_{\bar{\nu}}$ from one, is $10 \%$. The orbits for this case are fairly irregular. See Figures $8 \mathrm{a}$ and $8 \mathrm{~b}$.

\section{Relation to Behaviors Observed in the Early Universe}

In a certain region of parameter space for $\Delta<0$, ref. [9] observed irregular flavor behavior. It is interesting to ask whether this could be bimodal self-maintained coherence. The equations governing neutrino oscillations in the early Universe [6, (7) differ from those in Eq. (1.7) in two repects: First, there are CP-asymmetric effects. This means that the medium-induced linear term, the analog of $\vec{\Delta}$ in Eq. (1.8) is slightly different for neutrinos and antineutrinos. Second, $\vec{B}_{v}^{j}$ and $\vec{B}_{w}^{k}$ are time dependent due to the expansion of the Universe. However, in much of parameter space, interaction rates are faster than the expansion rate of the Universe, so that for short times $\vec{B}_{v}^{j}$ and $\vec{B}_{w}^{k}$ can be considered constant. Over long times, however, effects due to a non-constant Hubble constant can be important.

The CP-asymmetric effects, which are due to the excess of electrons over positrons, are relatively small. However, they can eventually lead to a situation in which antineutrino vectors are not exactly aligned with neutrino vectors. Since the neutrino gas is extremely dense, small CP-asymmetries in the neutrino sector may lead to a sizeable interaction in $\vec{V}_{\nu \nu}$. Hence, the electron-positron asymmetry might be impor- 
tant indirectly via the nonlinear neutrino-neutrino term.

Given this idea, it is reasonable to assume that the simplified model in Sect. II can represent the coherent behaviors for the early Universe, for the case where the energy distributions of neutrinos and antineutrinos are the same. The main effect of the positron-electron asymmetry is to misalign $\vec{y}$ and $\vec{z}$. Hence, it is of interest to examine solutions to Eq. (2.3) with $E_{0}=\bar{E}_{0}, n_{\nu}=n_{\bar{\nu}}$ and $\left|\vec{r}_{v}\right|=\left|\vec{r}_{w}\right|$, but with $\vec{r}_{v}(0) \neq \vec{r}_{w}(0)$.

We perform the same change of variables as in Eqs. (3.3) and (3.4). The CPasymmetric effects are then represented as a change in the initial conditions for $\vec{z}$ via

$$
\vec{z}(0)=\frac{n_{\nu}}{n_{\bar{\nu}}}(\cos (2 \theta-2 \alpha), \sin (2 \theta-2 \alpha) \cos 2 \beta, \sin (2 \theta-2 \alpha) \sin 2 \beta),
$$

while keeping $\vec{y}(0)$ as in Eq. (3.7), so that $\vec{y}(0)=(\cos 2 \theta, \sin 2 \theta, 0)$. Here, $\alpha$ and $\beta$ are angles that control the CP-asymmetry in the neutrino sector in the 1-2 plane and in the 3 direction.

Figure 9 displays the orbits for the neutrinos and antineutrinos for $\sin 2 \theta=0.8$, $\sin 2 \alpha=0.01, \sin 2 \beta=0.0, \gamma=\bar{\gamma}=0.1$ and $n_{\nu} / n_{\bar{\nu}}=1.0$. The motion is mostly back-and-forth as in the CP-symmetric case of reference [21] but the second mode causes the back-and-forth cycle to shift somewhat and not retrace over itself. When both $\alpha$ and $\beta$ are non-zero, fairly irregular behavior can arise. See Figure 10 which displays the orbits for the neutrinos and antineutrinos for $\sin 2 \theta=0.8, \sin 2 \alpha=0.1$, $\sin 2 \beta=0.1$, and $\gamma=\bar{\gamma}=0.1$. These examples show that bimodal self-maintained coherence and irregular flavor dependence arise even when the only CP-asymmetry is in the initial conditions for neutrinos and antineutrinos.

We have examined some of the orbits in the simulations of refs. [9, 11] to see whether bimodal coherence occurs in the early Universe. Due to the variety of manifestations of bimodal solutions, as typified in Figures 1-10, it is not always so easy to be sure that the irregular behavior seen corresponds to bimodal coherence. However, many results from oscillations in the early Universe are similar to the examples considered here. One clear case from ref. [11] occurs for the simulations with $\Delta=-1.0 \times 10^{-4}$ $\mathrm{eV}^{2}, \sin ^{2} 2 \theta=0.49$ at approximately 0.13 seconds after the Big Bang. Figure 11 dis- 
plays the orbit for the neutrinos in the flavor basis. The orbit for antineutrinos is virtually the same because of the CP-suppression mechanism discovered in refs. [6, 7]. Two modes are clearly seen. The orbit in Figure 11 is qualitatively similar to the orbit in Figure 2. Another example from ref. [11] occurs for $\Delta=-1.0 \times 10^{-6} \mathrm{eV}^{2}$, $\sin ^{2} 2 \theta=1.0 \times 10^{-8}$ at approximately 0.94 seconds. Although we do not display the orbit, it is similar to Figure 9a.

Bimodal self-maintained coherence might be the general behavior of many dense neutrino gas systems. All three behaviors observed in the simulations of refs. [0, 9, 11] are qualitatively obtained as limits. Irregular behavior corresponds to bimodal coherence when both modes have sizeable amplitudes. Residual self-maintained coherence occurs when only one mode has a sizeable amplitude. Finally, smooth behavior arises when vectors point in the directions of the the linear terms in the effective magnetic fields, so that both modes have small amplitudes. Indeed, for a dense neutrino gas in the early Universe, tiny rapid fluctuations were observed in the simulations of refs. [6, 7, 9, 10, 11] in the smooth region of the phase diagrams. One possible explanation for them is that they correspond to small-amplitude bimodal coherence. Of course, when the amplitudes for oscillations are tiny, other effects may create them. Even if all the behaviors in parameter space for $\Delta<0$ correspond to bimodal coherence, it is still useful to characterize them by irregular, residual self-maintained coherence, and smooth as in Figure 1 of ref. [9].

\section{Neutrino Behavior in the Early Universe for $\mu_{\nu} \neq 0$}

The studies of neutrino oscillations in the early Universe in refs. [6, 7, 9, 10, 11] were performed under the assumption that $n_{\nu}=n_{\bar{\nu}}$ and that the energy distributions for neutrinos and for antineutrinos are the same. Given that there is a CP-asymmetry for charged leptons, namely the excess of electrons over positrons, it is possible that the numbers of neutrinos and antineutrinos are not equal, in which case a chemical potential potential $\mu_{\nu}$ for neutrinos needs to be introduced.

One purpose of the current work is to gain insight into the asymmetric neutrinoantineutrino gas. With this insight, it is possible to speculate on the phase diagram 
and on the flavor behavior. To rigorously determine these, simulations must be performed, but such computer studies are numerically intensive because one must explore a phase space of three parameters: $\theta, \Delta$ and $\mu_{\nu}$. A thorough study cannot be undertaken with the power of present computers. However, an educated guess as to the results can be made.

Due to the explicit asymmetry between neutrinos and antineutrinos when $\mu_{\nu} \neq 0$, residual self-maintained coherence, which corresponds to a situation in which only a single mode is excited, is unlikely to appear, except for perhaps brief periods for exceptional values of parameters. Instead, irregular flavor, as governed by bimodal coherence, should replace it. Thus the diagram for $\Delta<0$ probably has only two phases: smooth and irregular. When $\mu_{\nu} \neq 0$, irregular behavior should arise in the regions of the phase diagrams of refs. [9, 11] where irregular or residual self-maintained coherence occurred.

For $\Delta>0$, it is likely that both neutrino and antineutrino vectors follow their respectively effective magnetic fields, thereby maintaining themselves in approximate nonlinear mass eigenstates. Hence, smooth behavior is generally expected for $\Delta>0$. However, if $\bar{E}_{0}$ is significantly different from $E_{0}$ then transitory weak irregular behavior should take place in the "cross-over region". The cross-over region is the period during which neutrinos evolve from approximate flavor eigenstates to approximate vacuum mass eigenstates [7]. Small amplitude irregular behavior should appear there because neutrinos and antineutrinos make the transition to mass eigenstates at different times. This cause neutrino and antineutrinos vectors to point in different directions. The effect should be most pronounced for small $\Delta$, particularly when $\Delta<10^{-9} \mathrm{eV}^{2}$. After the transition region, the behavior should become smooth again. A similar transitory effect might also occur in the smooth phase for $\Delta<0$.

One motivation for considering a non-zero neutrino chemical potential is that it introduces additional CP-asymmetry into the system. Reference [14 showed that a large neutrino-antineutrino asymmetry can have an effect on nucleosynthesis. However, for the values of the parameters of the early Universe, $\varphi$-phase locking is expected. This implied that the CP-asymmetry due to $\mu_{\nu} \neq 0$ should not be amplified in time. Hence, neutrino oscillations for $\mu_{\nu} \neq 0$ are likely to affect nucleosynthesis 
at the same level as in the case of no neutrino masses and mixing [22, 23]. Constraints on the chemical potential of neutrinos from Big Bang nucleosynthesis have been obtained in ref. [16.

\section{Summary}

In this work, we analyzed neutrino oscillations in a dense gas in which there is an explicit neutrino-antineutrino asymmetry. The energy distribution for antineutrinos was not necessarily the same as the energy distribution for neutrinos, nor did the numbers of antineutrinos and neutrinos have to be the same.

In the dense gas limit, alignment is expected to hold. Using this property, the nonlinear multi-equation system was simplified to six equations in Section II. Remarkably, the six nonlinear equations were solvable. The solution was still quite complicated. After performing some transformations in Section III, most solution details were relegated to Appendix A. The generic behavior was bimodal self-maintained coherence. This is a collective soliton-like solution in which neutrino behavior is cooperative and in which two modes of different frequencies are present.

Bimodal self-maintained coherence exhibits a wide range of behaviors depending on parameters and initial conditions. Particular illustrative cases were presented in Section IV.

When the amplitude for one of the modes vanishes, ordinary self-maintained coherence is obtained. Such behavior was first observed in test simulations in ref. [1. It was subsequently observed in numerical simulations of the early Universe in ref. [9] and understood analytically in ref. [21]. Another neutrino flavor behavior, which has been seen in the early Universe, is smooth [6, 7]. It corresponds to a bimodal solution in which the amplitudes of both modes are small. Finally, irregular behavior, which can occur for an inverted mass hierarchy [9], is mimicked by bimodal coherence when both amplitudes are present. These results suggest that bimodal coherence may be the general flavor behavior for many dense neutrino gases. As discussed in Section $\mathrm{V}$, our results provide new insight into the simulations of neutrino oscillations in the early Universe performed in refs. [6, 7, 9, 10, 11]. 
With this additional understanding, it was possible to surmise the neutrino flavor dependence in the early Universe when neutrinos have a chemical potential, so that there is an explicit neutrino-antineutrino asymmetry. The expected behavior for $\Delta>0$ is smooth with a possible weak bimodal coherent phase for a short time period. The expected behaviors for the inverted mass hierarchy case, $\Delta<0$, are smooth and bimodal coherence. Bimodal coherence probably arises where irregular or residual self-maintained coherence arose for the $\mu_{\nu}=0$ case of refs. [9, 11]. Because of the phenomenon of $\varphi$-phase locking, effects on nucleosynthesis are not expected to be amplified by neutrino oscillations, as one might have a priori thought. The effects are probably limited to those directly due to a non-zero neutrino chemical potential [16].

In short, our work has shown that dense neutrino gases can exhibit interesting and non-intuitive phenomenon. The gases often have collective, cooperative and coherent flavor behavior.

\section{Acknowledgements}

I would like to thank Alan Kostelecký for discussions. I also thank Julius Wess for hospitality. This work is supported in part by the United States Department of Energy (grant number DE-FG02-92ER40698), by the Alexander von Humboldt Foundation, and by the PSC Board of Higher Education at CUNY.

\section{Appendix A}

It turns out that Eq. (3.5) can be solved for arbitrary initial conditions. Four conserved quantities play a role. The first two

$$
y_{1}^{2}+y_{2}^{2}+y_{3}^{2}=1
$$

and

$$
z_{1}^{2}+z_{2}^{2}+z_{3}^{2}=\left(\frac{n_{\nu}}{n_{\bar{\nu}}}\right)^{2}
$$

correspond to conservation of neutrinos and of antineutrinos. Equation (3.5) also implies that

$$
y_{1}-z_{1}=k_{1}
$$


and that

$$
y_{1}-\frac{1}{2 \gamma(\bar{\gamma}+\gamma)}\left(\left(y_{2}-z_{2}\right)^{2}+\left(y_{3}-z_{3}\right)^{2}\right)=k_{12}
$$

where $k_{1}$ and $k_{12}$ are time-independent constants. They are determined by initial conditions, i.e.,

$$
\begin{gathered}
k_{1}=y_{1}(0)-z_{1}(0) \\
k_{12}=y_{1}(0)-\frac{1}{2 \gamma(\bar{\gamma}+\gamma)}\left(\left(y_{2}(0)-z_{2}(0)\right)^{2}+\left(y_{3}(0)-z_{3}(0)\right)^{2}\right)
\end{gathered}
$$

For initial flavor eigenstates,

$$
\begin{gathered}
k_{1} \rightarrow \cos 2 \theta\left(1-\frac{n_{\nu}}{n_{\bar{\nu}}}\right) \\
k_{12} \rightarrow \cos 2 \theta-\frac{\sin ^{2} 2 \theta}{2 \gamma(\bar{\gamma}+\gamma)}\left(1-\frac{n_{\nu}}{n_{\bar{\nu}}}\right)^{2} .
\end{gathered}
$$

It turns out to be useful to solve for the quantity $y_{2} z_{2}+y_{3} z_{3}$ using Eqs. (A.1)-(A.4). One finds

$$
y_{2} z_{2}+y_{3} z_{3}=\frac{1}{2}\left(1-k_{1}^{2}+\left(\frac{n_{\nu}}{n_{\bar{\nu}}}\right)^{2}\right)+\gamma(\bar{\gamma}+\gamma)\left(k_{12}-y_{1}\right)+k_{1} y_{1}-y_{1}{ }^{2} .
$$

Thus, Eqs. (A.1)-(A.4) allow one to eliminate four combinations of variables in favor of $y_{1}$ :

$$
\begin{gathered}
y_{2}{ }^{2}+y_{3}{ }^{2} \rightarrow 1-y_{1}{ }^{2} \\
z_{1} \rightarrow y_{1}-k_{1} \\
z_{2}^{2}+z_{3}^{2} \rightarrow\left(\frac{n_{\nu}}{n_{\bar{\nu}}}\right)^{2}-\left(y_{1}-k_{1}\right)^{2} \\
y_{2} z_{2}+y_{3} z_{3} \rightarrow \frac{1}{2}\left(1-k_{1}{ }^{2}+\left(\frac{n_{\nu}}{n_{\bar{\nu}}}\right)^{2}\right)+\gamma(\bar{\gamma}+\gamma)\left(k_{12}-y_{1}\right)+k_{1} y_{1}-y_{1}{ }^{2} .
\end{gathered}
$$

Take the first order differential equation for $y_{1}$ in Eq. (3.5), differentiate it with respect to $\tau$ and use the equations for the second and third components in Eq. (3.5). It is a miracle that precisely the four quantities on the left-hand side of Eq. (A.8) enter. Straightforward but lengthy algebra gives

$$
\frac{d^{2} y_{1}}{d \tau^{2}}=\left(1+\frac{\bar{\gamma}}{\gamma}\right)\left(y_{2} z_{2}+y_{3} z_{3}\right)+
$$




$$
\begin{gathered}
\frac{\left(y_{1}+z_{1}\right)\left(y_{2} z_{2}+y_{3} z_{3}\right)-y_{1}\left(z_{2}^{2}+z_{3}^{2}\right)-\left(y_{2}^{2}+y_{3}^{2}\right) z_{1}}{\gamma^{2}} \\
=-3 \hat{A} y_{1}^{2}-2 \hat{B} y_{1}-\hat{C}
\end{gathered}
$$

where, in the last equality, the substitutions in Eq. (A.8) are used. The constants $\hat{A}$, $\hat{B}$ and $\hat{C}$ are given by

$$
\begin{gathered}
\hat{A}=\frac{\bar{\gamma}+\gamma}{\gamma}, \\
\hat{B}=\frac{(\bar{\gamma}+\gamma)^{2}}{2}+\frac{k_{1}^{2}}{2 \gamma^{2}}-\frac{(\bar{\gamma}+\gamma)\left(k_{1}+k_{12}\right)}{\gamma} \\
\hat{C}=-(\bar{\gamma}+\gamma)^{2} k_{12}-\frac{k_{1}}{2 \gamma^{2}}\left(1+k_{1}^{2}-\left(\frac{n_{\nu}}{n_{\bar{\nu}}}\right)^{2}\right) \\
+\frac{(\bar{\gamma}+\gamma)}{\gamma}\left(k_{1} k_{12}-\frac{1}{2}\left(1-k_{1}{ }^{2}+\left(\frac{n_{\nu}}{n_{\bar{\nu}}}\right)^{2}\right)\right)
\end{gathered}
$$

Thus the motion of $y_{1}$ corresponds to a classical particle moving in a one-dimensional cubic potential. This system can be solved by quadratures. Multiply Eq. (A.9) by $\frac{d y_{1}}{d \tau}$ and integrate to obtain

$$
\frac{1}{2}\left(\frac{d y_{1}}{d \tau}\right)^{2}=-V\left(y_{1}\right)
$$

where

$$
V\left(y_{1}\right)=-\frac{1}{2}\left(\frac{d y_{1}}{d \tau}(0)\right)^{2}+\hat{C}\left(y_{1}-y_{1}(0)\right)+\hat{B}\left(y_{1}^{2}-\left(y_{1}(0)\right)^{2}\right)+\hat{A}\left(y_{1}^{3}-\left(y_{1}(0)\right)^{3}\right) .
$$

The potential $V\left(y_{1}\right)$ has three zeroes and is negative between the two larger zeroes. The motion of $y_{1}$ is between these two larger zeroes where $V\left(y_{1}\right) \leq 0$. Integration of Eq. (A.11) leads to

$$
\tau=\int_{y_{1}(0)}^{y_{1}(\tau)} \frac{d y}{\sqrt{-2 V(y)}} .
$$

This equation gives $\tau$ in terms of $y_{1}(\tau)$ where the latter appears as the upper limit of integration. Equation (A.13) is inverted to obtain $y_{1}$ as a function of $\tau$.

Since the potential is cubic, the solution is always related to Jacobi elliptic functions. In general, a cubic equation must be solved, so that the explicit solution is rather complicated. However, when

$$
\frac{d y_{1}}{d \tau}(0)=0
$$


one can avoid this difficulty. The initial condition in Eq. (A.14) is an important case because it includes the initial flavor eigenstates in Eq. (3.7). When Eq. (A.14) holds, the potential becomes!

$$
V\left(y_{1}+y_{1}(0)\right)=y_{1}\left(A y_{1}^{2}+B y_{1}+C\right)=\frac{\bar{\gamma}+\gamma}{\gamma} y_{1}\left(y_{1}-y_{+}\right)\left(y_{1}+y_{-}\right)
$$

where

$$
\begin{gathered}
A=\hat{A} \\
B=\hat{B}+3 \hat{A} y_{1}(0) \\
C=\hat{C}+2 \hat{B} y_{1}(0)+3 \hat{A}\left(y_{1}(0)\right)^{2} .
\end{gathered}
$$

The zeroes of the potential are $y_{1}(0)-y_{-}, y_{1}(0)$ and $y_{1}(0)+y_{+}$where

$$
y_{+}=\frac{-B+\sqrt{B^{2}-4 A C}}{2 A}, \quad y_{-}=\frac{B+\sqrt{B^{2}-4 A C}}{2 A}
$$

Thus $y_{1}$ oscillates between $y_{1}(0)$ and $y_{1}(0)+y_{+}$.

The integral in Eq. (A.13) is

$$
y_{1}^{s o l}(\tau)=y_{1}(0)+\frac{y_{-} y_{+}}{y_{0}} \frac{s n^{2}\left(\tau \sqrt{\frac{y_{0}(\bar{\gamma}+\gamma)}{2 \gamma}}, \frac{y_{+}}{y_{0}}\right)}{d n^{2}\left(\tau \sqrt{\frac{y_{0}(\bar{\gamma}+\gamma)}{2 \gamma}}, \frac{y_{+}}{y_{0}}\right)},
$$

where

$$
y_{0} \equiv \frac{\sqrt{B^{2}-4 A C}}{A}=y_{+}+y_{-}
$$

and where $s n$ and $d n$ are the sine-amplitude and delta-amplitude Jacobi elliptic functions.

Hence $y_{1}$ undergoes periodic motion with a period $\mathcal{T}_{f}$ in $\tau$ given by

$$
\mathcal{T}_{f}=2 \sqrt{\frac{2 \gamma}{y_{-}(\bar{\gamma}+\gamma)}} K\left(-q^{2}\right),
$$

where

$$
K\left(-q^{2}\right)=\int_{0}^{1} \frac{d w}{\sqrt{\left(1-w^{2}\right)\left(1+q^{2} w^{2}\right)}},
$$

\footnotetext{
1 For the case of general initial conditions one must shift $y_{1}$ by a constant which renders the middle zero at 0 . Then one proceeds as in the special case explicitly carried out here.
} 
with $q^{2} \equiv y_{+} / y_{-}$. The period $T_{f}$ in $t$ is

$$
T_{f}=2 \frac{E_{0}}{\Delta} \sqrt{\kappa_{0}} \mathcal{T}_{f}
$$

The solution for $z_{1}(\tau)$ is given by using Eqs. (A.3) and (A.18).

Finding the solution of the second and third components of $\vec{y}$ and $\vec{z}$ is not so straightforward. One proceeds by using the polar coordinate angles in the two-three plane, i.e., let $\varphi=\tan ^{-1}\left(y_{3} / y_{2}\right)$ and $\bar{\varphi}=\tan ^{-1}\left(z_{3} / z_{2}\right)$. Differentiate $\varphi$ with respect to $\tau$ and use Eq. (3.5). Do the same for $\bar{\varphi}$. Miraculously, only the four quantities in Eq. (A.8) enter. Hence, the substitutions in Eq. (A.8) can be used to express $\frac{d \varphi}{d \tau}$ and $\frac{d \bar{\varphi}}{d \tau}$ in terms of $y_{1}(\tau)$. After straightforward but lengthy algebra, one finds

$$
\begin{gathered}
\frac{d \varphi}{d \tau}=-\gamma+\frac{k_{1}+\left(\gamma(\bar{\gamma}+\gamma)\left(k_{12}-y_{1}\right)-\frac{1}{2}\left(1+k_{1}^{2}-\left(\frac{n_{\nu}}{n_{\bar{\nu}}}\right)^{2}\right)\right) y_{1}}{\gamma\left(1-y_{1}^{2}\right)}, \\
\frac{d \bar{\varphi}}{d \tau}=\bar{\gamma}+\frac{\left(\frac{1}{2}\left(1-k_{1}^{2}+\left(\frac{n_{\nu}}{n_{\bar{\nu}}}\right)^{2}\right)+\gamma(\bar{\gamma}+\gamma)\left(k_{12}-y_{1}\right)\right)\left(k_{1}-y_{1}\right)+\left(\frac{n_{\nu}}{n_{\bar{\nu}}}\right)^{2} y_{1}}{\gamma\left(\left(\frac{n_{\nu}}{n_{\bar{\nu}}}\right)^{2}-\left(y_{1}-k_{1}\right)^{2}\right)} .
\end{gathered}
$$

Thus,

$$
\varphi\left(\tau^{\prime}\right)=\varphi(0)+\int_{0}^{\tau^{\prime}} d \tau \frac{d \varphi}{d \tau}, \quad \bar{\varphi}\left(\tau^{\prime}\right)=\bar{\varphi}(0)+\int_{0}^{\tau^{\prime}} d \tau \frac{d \bar{\varphi}}{d \tau}
$$

where $\frac{d \varphi}{d \tau}$ and $\frac{d \bar{\varphi}}{d \tau}$ are given in Eq. (A.23) and where

$$
\varphi(0)=\tan ^{-1}\left(\frac{y_{3}(0)}{y_{2}(0)}\right), \quad \bar{\varphi}(0)=\tan ^{-1}\left(\frac{z_{3}(0)}{z_{2}(0)}\right) .
$$

The solutions for the second and third components are then given via Eqs. (3.8) and (3.9).

Since $y_{1}$ is periodic, the angular motion in the two-three plane is also periodic but with a period different from $\mathcal{T}_{f}$. For neutrinos, let $\mathcal{T}_{s}$ denote the period in $\tau$. For antineutrinos, let $\overline{\mathcal{T}_{s}}$ be the period. Then,

$$
\mathcal{T}_{s}=\frac{2 \pi \mathcal{T}_{f}}{\left|\int_{0}^{\mathcal{T}_{f}} d \tau \frac{d \varphi}{d \tau}\right|}, \quad \overline{\mathcal{T}}_{s}=\frac{2 \pi \mathcal{T}_{f}}{\left|\int_{0}^{\mathcal{T}_{f}} d \tau \frac{d \bar{\varphi}}{d \tau}\right|}
$$


where || indicates absolute value. The subscripts $f$ and $s$ on $\mathcal{T}_{f}$ and $\mathcal{T}_{s}$ stands for "fast" and "slow". In the extreme dense gas limit $\mathcal{T}_{f}$ is much smaller than $\mathcal{T}_{s}$, i.e., $\mathcal{T}_{f}$ is the period for the faster motion. The periods $T_{s}$ and $\bar{T}_{s}$ in terms of $t$ are

$$
T_{s}=2 \frac{E_{0}}{\Delta} \sqrt{\kappa_{0}} \mathcal{T}_{s}, \quad \bar{T}_{s}=2 \frac{E_{0}}{\Delta} \sqrt{\kappa_{0}} \overline{\mathcal{T}}_{s}
$$

\section{References}

1. See, for example, E. W. Kolb and M.S. Turner, The Early Universe (Addison Wesley, Redwood City, CA 1990).

2. See, for example, C. W. Kim and A.Pevsner, Neutrinos in Physics and Astrophysics (Harwood, Langhorne, PA, 1993).

3. See, for example, J.Bahcall, Neutrino Astrophysics (Cambridge University Press, Cambridge, 1989).

4. S. Samuel, Phys. Rev. D48 (1993) 1462.

5. G. Sigl and G. Raffelt, Nucl. Phys. B406 (1993) 423.

6. V. A. Kostelecký, J. Pantaleone and S. Samuel, Phys. Lett. B315 (1993) 46.

7. V. A. Kostelecký and S. Samuel, Phys. Rev. D49 (1994) 1740.

8. Y.-Z. Qian and G. M.Fuller, Phys. Rev. D51 (1995) 1479.

9. V. A. Kostelecký and S. Samuel, Phys. Lett. B318 (1993) 127.

10. V. A. Kostelecký and S. Samuel, Phys. Rev. D52 (1995) 3184.

11. V.A. Kostelecký and S. Samuel, Nonequilibrium Neutrino Oscillations in the Early Universe with an Inverted Neutrino-Mass Hierarchy, CCNY-HEP-96/1, IUHET 326, work in progress to appear.

12. A. D. Dolgov, Sov. J. Nucl. Phys. 33 (1981) 700.

13. L. Stodolsky, Phys. Rev. D36 (1987) 2272.

14. P. Langacker, S. T. Petcov, G. Steigman, and S. Toshev, Nucl. Phys. B282 (1987) 589.

15. D. Notzold and G. Raffelt, Nucl. Phys. B307 (1988) 924. 
16. M. J. Savage, R. A. Malaney and G. M. Fuller, Astrophys. J. 368 (1991) 1.

17. K. Enqvist, K. Kainulainen and J. Maalampi, Nucl. Phys. B349 (1991) 754.

18. K. Enqvist, K. Kainulainen and M. Thomson, Nucl. Phys. B373 (1992) 498.

19. X. Shi, D. N. Schramm and B. D. Fields, Phys. Rev. D48 (1993) 2563.

20. B. H. J. McKellar and M. J. Thomson Phys. Rev. D49 (1994) 2710.

21. V. A. Kostelecký and S. Samuel, Phys. Rev. D52 (1995) 621.

22. S. Dodelson and M. S. Turner, Phys. Rev. D46 (1992) 3372;

B. D. Fields, S. Dodelson and M. S. Turner, Phys. Rev. D47 (1993) 4309.

23. A. D. Dolgov and M. Fukugita, Phys. Rev. D46 (1992) 5378.

\section{Figure Captions}

Figure 1. Components of $\vec{y}$ as a function of scaled time $\tau$ for $0<\tau<20$ for the case $\sin 2 \theta=0.8, n_{\nu} / n_{\bar{\nu}}=0.9, \gamma=0.1$ and $\bar{\gamma}=0.12$ and with initial conditions as in Eq. (3.7). (a) The component $y_{1}$. (b) The component $y_{2}$. (c) The component $y_{3}$.

Figure 2. The three-dimensional orbits for the example in Figure 1. (a) The neutrino orbit. (b) The antineutrino orbit.

Figure 3. The flavor component $r_{v 1}$ as a function of $\tau$. Parameters are the same as in Figure 1.

Figure 4. The orbits during $0<\tau<10$ for the case $\sin 2 \theta=0.8, n_{\nu} / n_{\bar{\nu}}=0.75$, $\gamma=0.1$ and $\bar{\gamma}=0.12$ and with initial conditions as in Eq. (3.7). (a) The neutrino orbit. (b) The antineutrino orbit.

Figure 5. A plot of $y_{2}$ versus $\tau$ for the example in Figure 4.

Figure 6. The orbits during for $0<\tau<20$ for the case $\sin 2 \theta=0.8, n_{\nu} / n_{\bar{\nu}}=0.4$, $\gamma=0.5$ and $\bar{\gamma}=0.6$ and with initial conditions as in Eq. (3.7). (a) The neutrino orbit. (b) The antineutrino orbit.

Figure 7. A comparison of $y_{2}$ and $z_{2}$ when $\varphi$-phase locking does not hold for the case $\sin 2 \theta=0.8, n_{\nu} / n_{\bar{\nu}}=0.9$ and $\gamma=\bar{\gamma}=0.5$. (a) The component $y_{2}$. (b) The component $z_{2}$. 
Figure 8. The orbits during $0<\tau<10$ for parameters as in Figure 7. (a) The neutrino orbit. (b) The antineutrino orbit.

Figure 9. The orbits during $0<\tau<10$ for the case $\sin 2 \theta=0.8, n_{\nu} / n_{\bar{\nu}}=1.0$ and $\gamma=\bar{\gamma}=0.1$ and using the initial conditions in Eq. (5.1) with $\sin 2 \alpha=0.01$ and $\sin 2 \beta=0.0$. (a) The neutrino orbit. (b) The antineutrino orbit.

Figure 10. The orbits during $0<\tau<10$ for the case $\sin 2 \theta=0.8, n_{\nu} / n_{\bar{\nu}}=1.0$ and $\gamma=\bar{\gamma}=0.1$ and using the initial conditions in Eq. (5.1) with $\sin 2 \alpha=\sin 2 \beta=0.1$. (a) The neutrino orbit. (b) The antineutrino orbit.

Figure 11. A neutrino orbit with axes in the flavor basis for $\Delta=-1.0 \times 10^{-6} \mathrm{eV}^{2}$ and $\sin ^{2} 2 \theta=1.0 \times 10^{-8}$ during a small time interval at approximately 0.13 seconds after the Big Bang. 


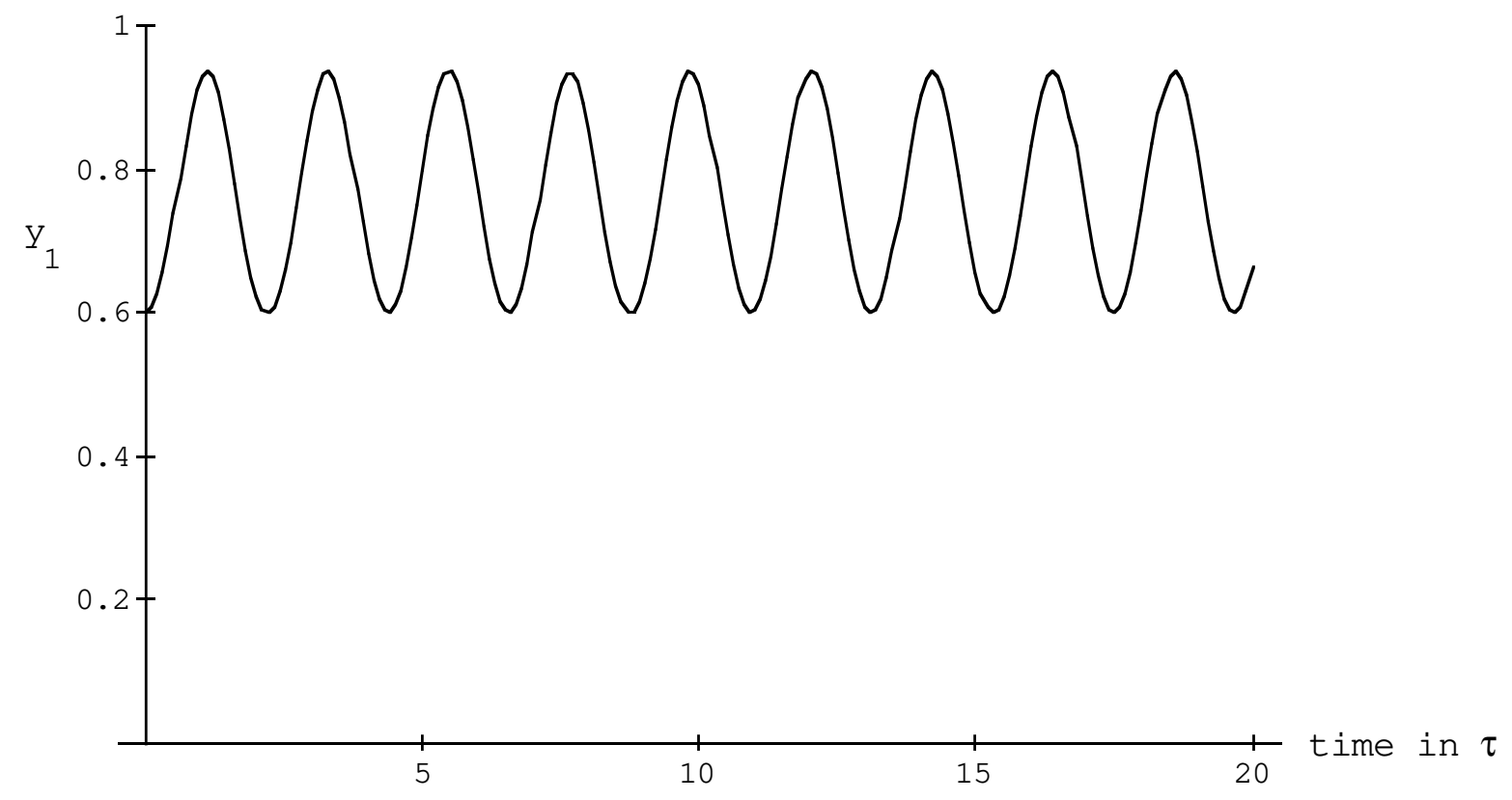

(a)

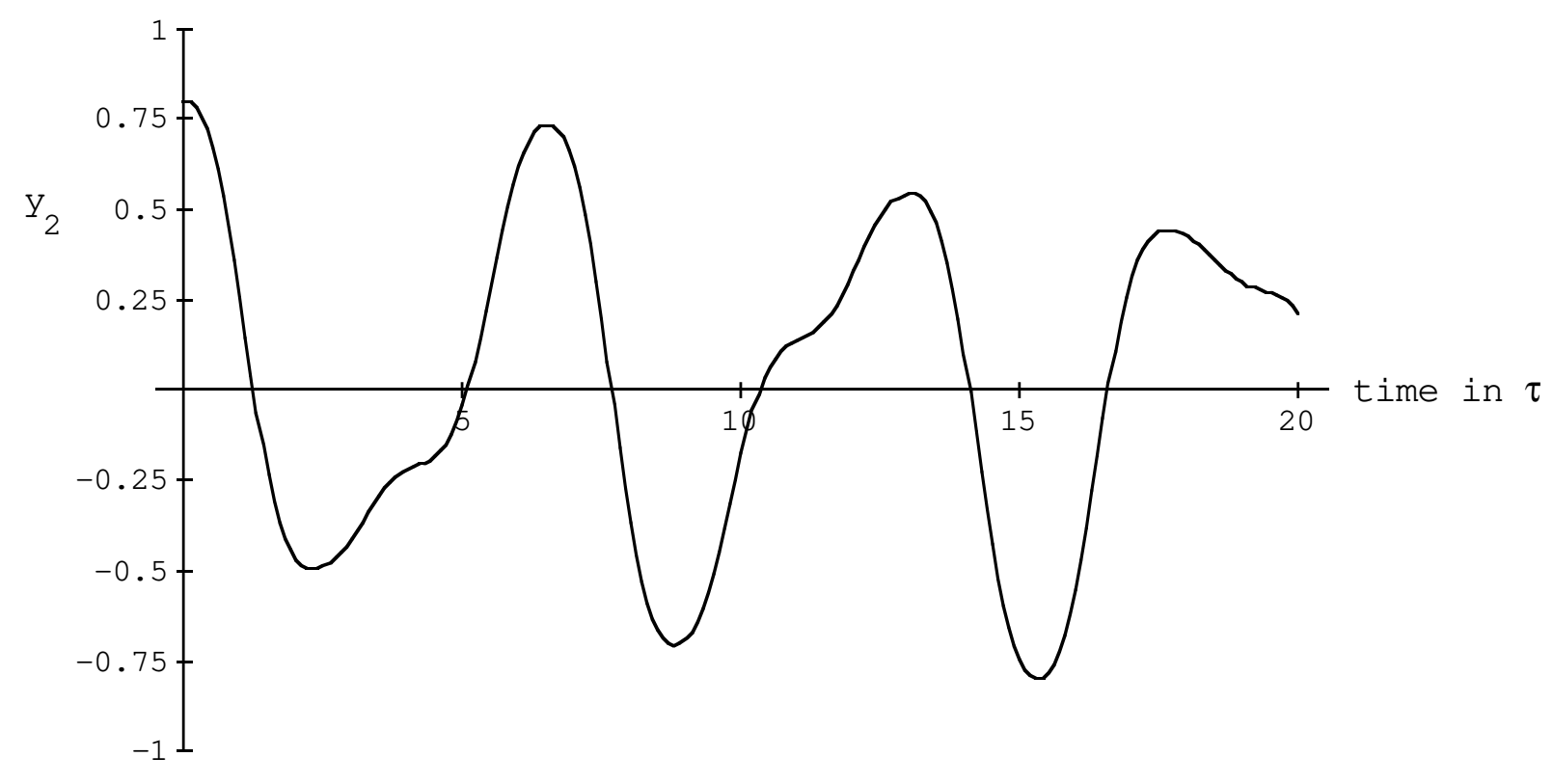

(b)

Figure 1 


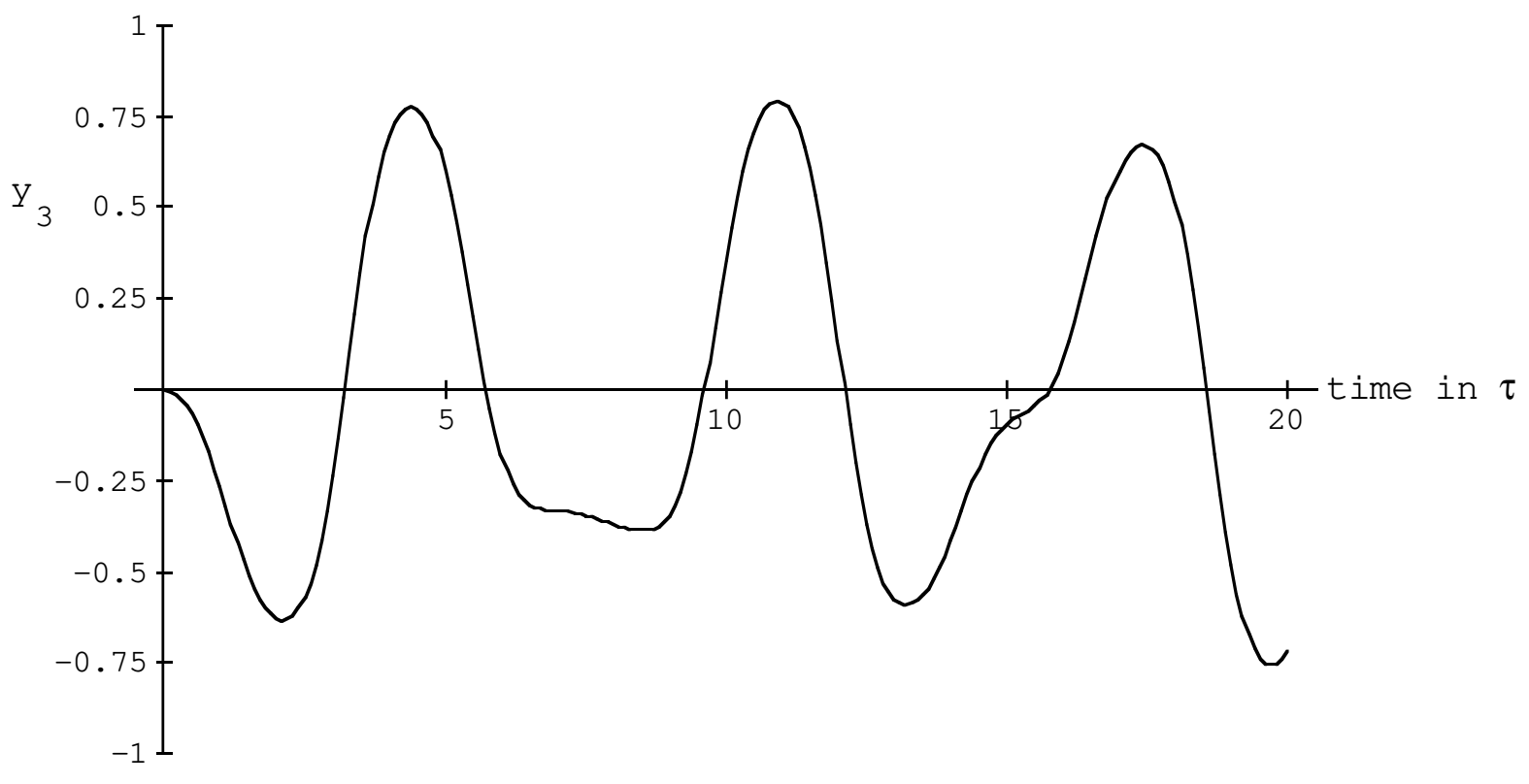

(c)

Figure 1 


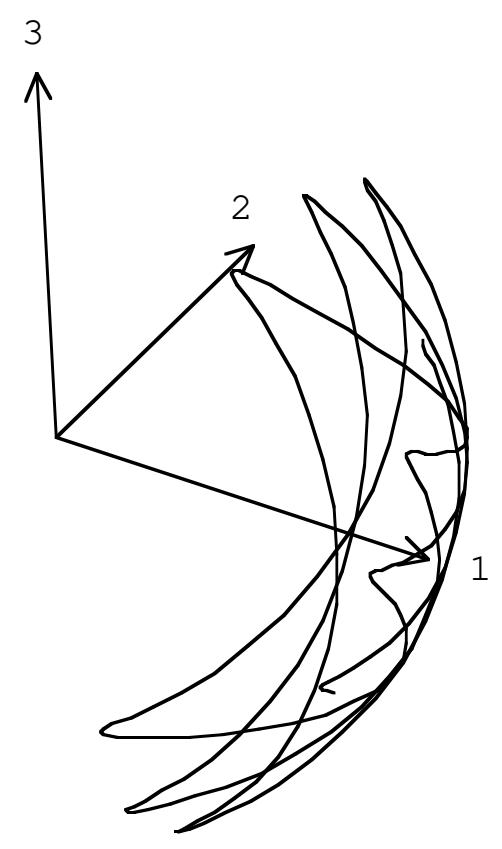

(a)

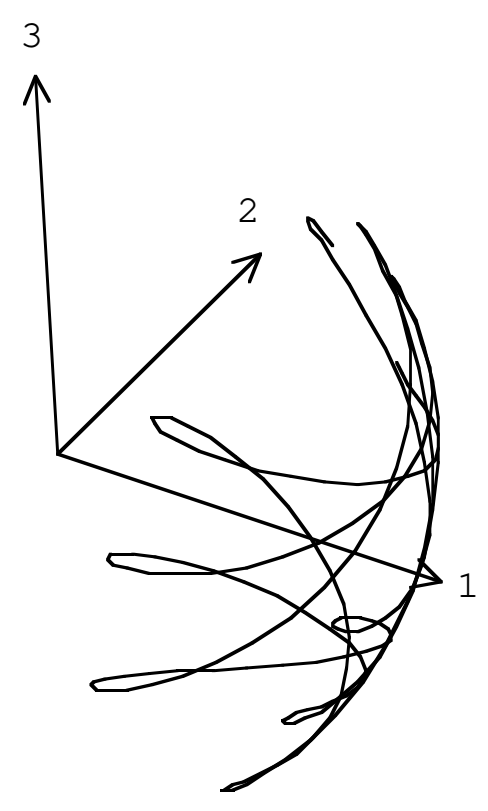

(b)

Figure 2 


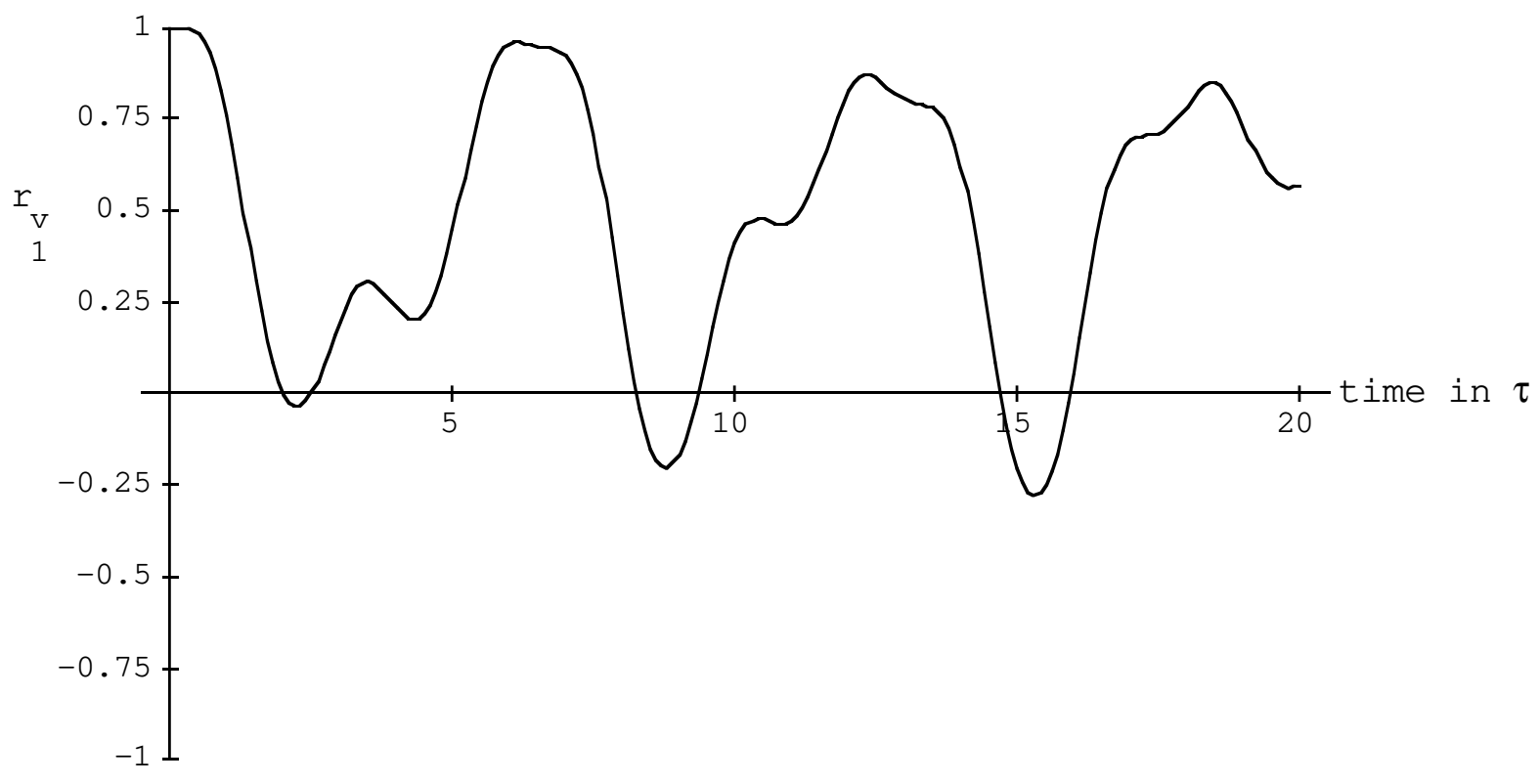

Figure 3 


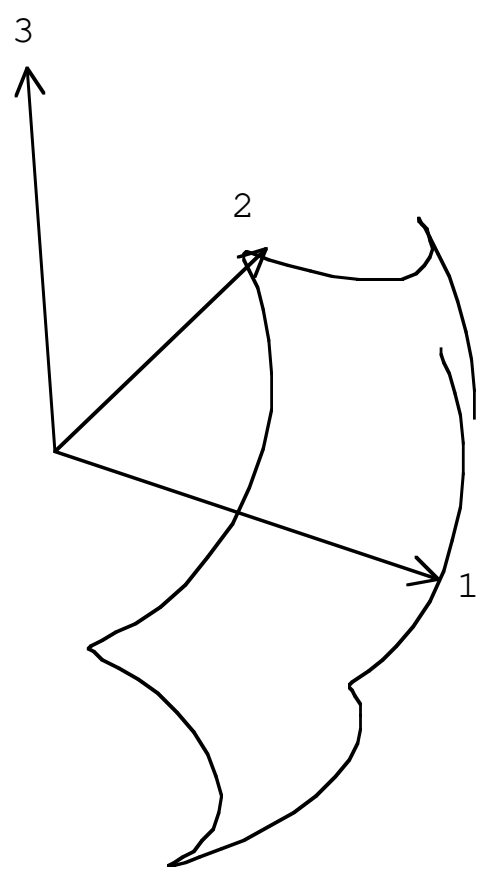

(a)

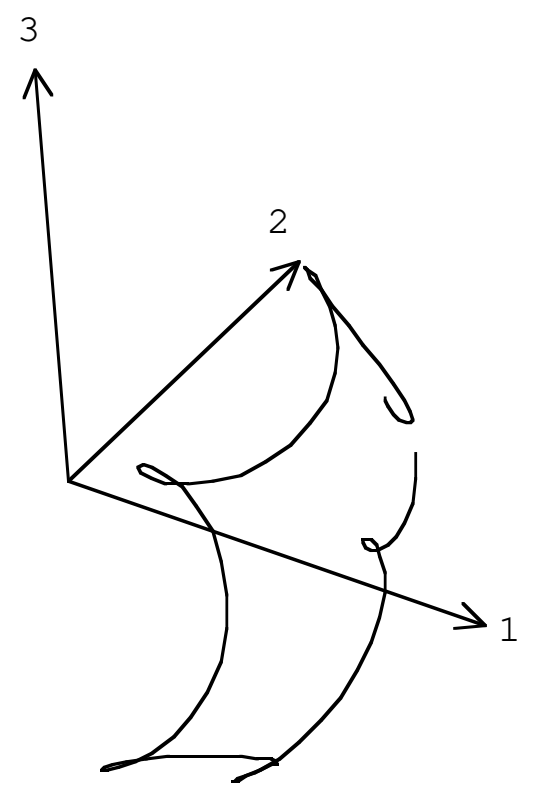

(b) 


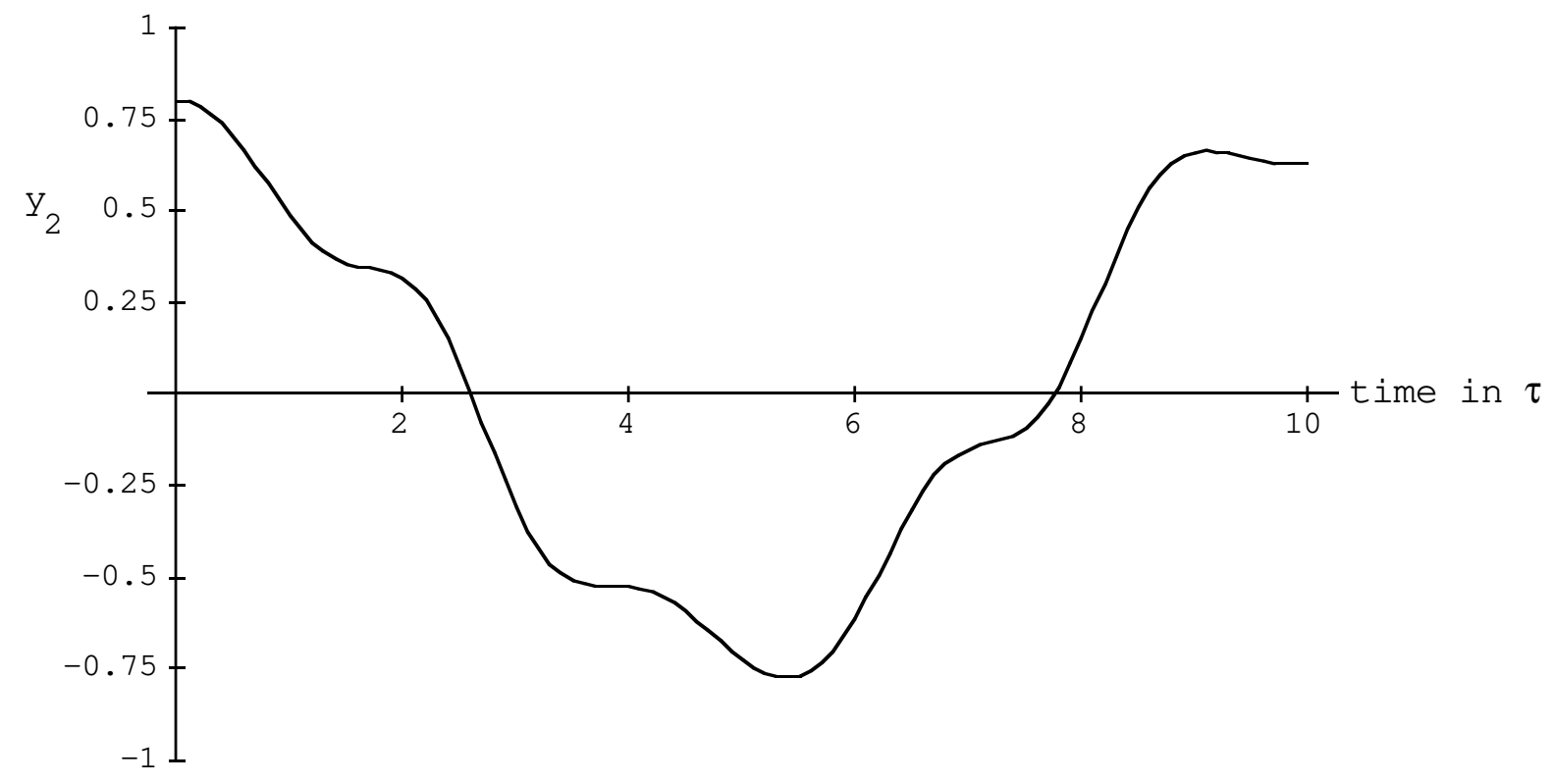

Figure 5 


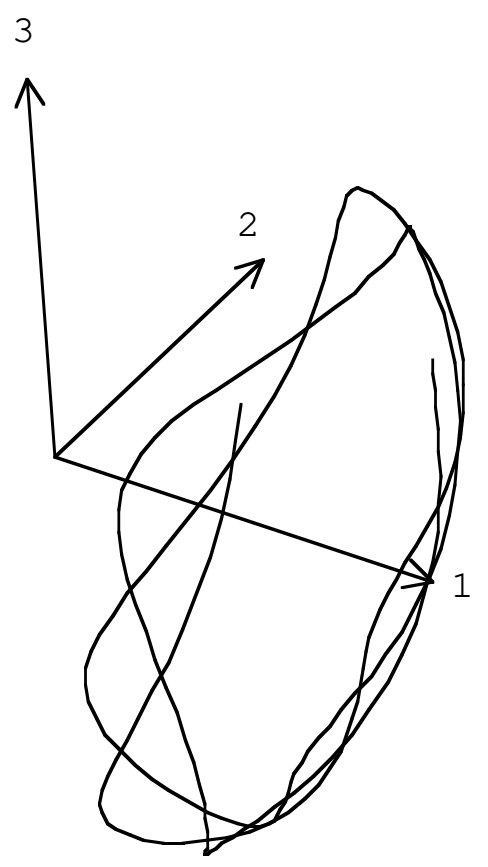

(a)

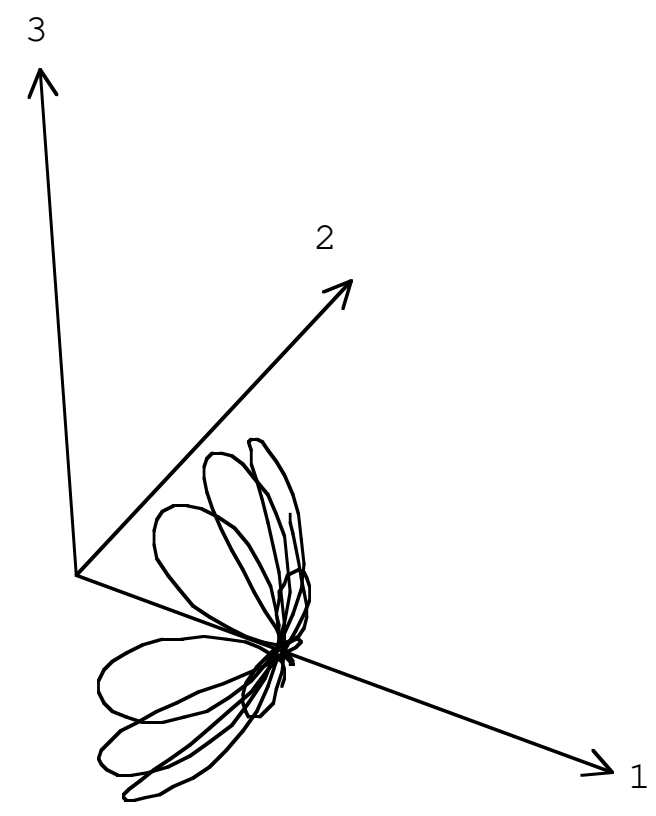

(b) 


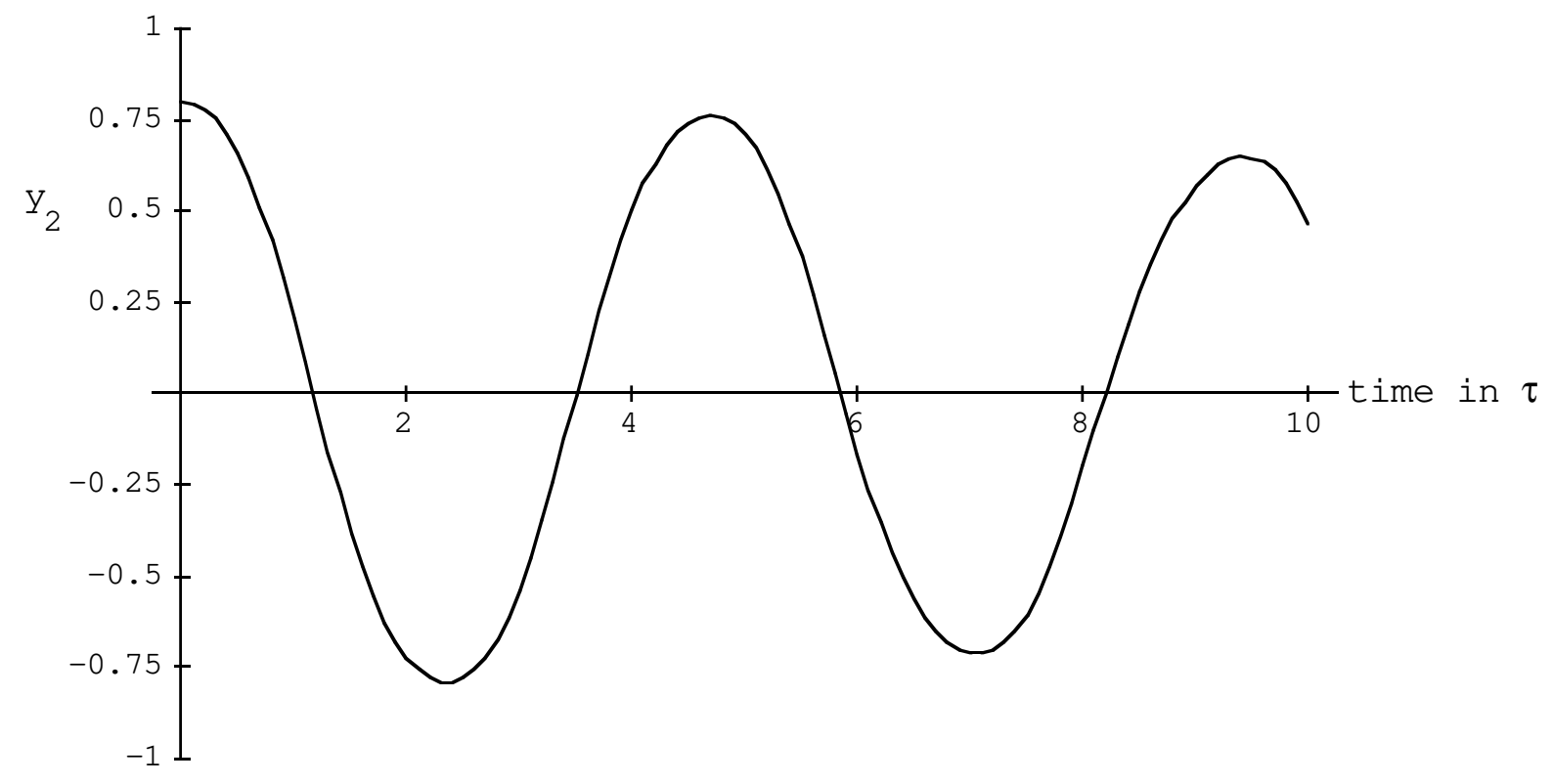

(a)

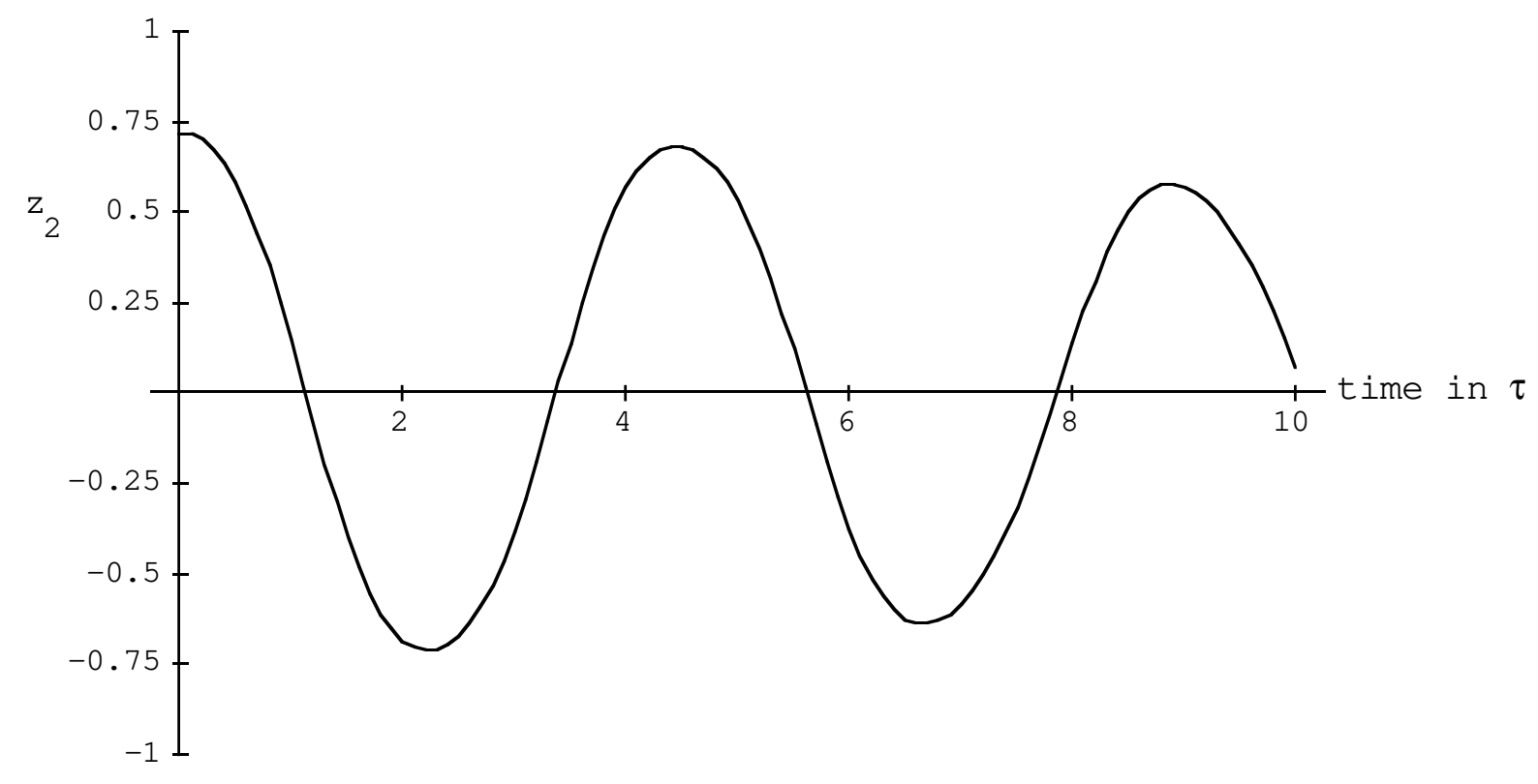

(b)

Figure 7 


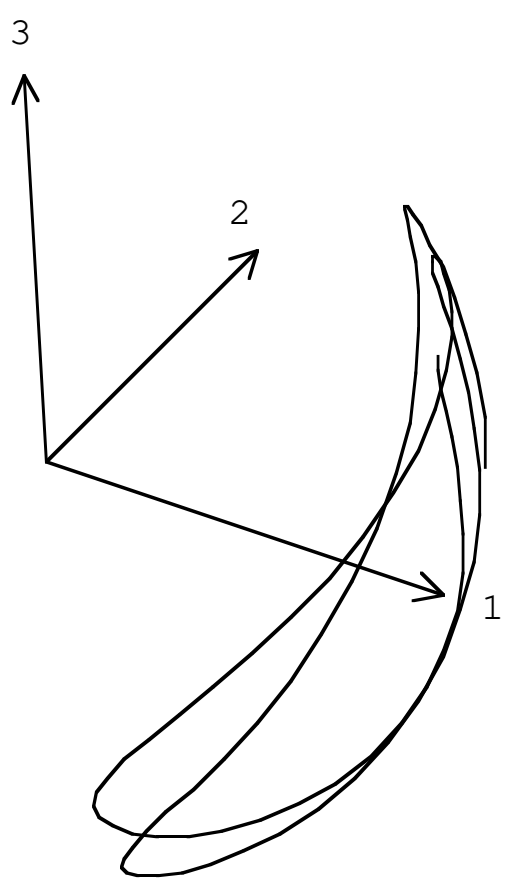

(a)

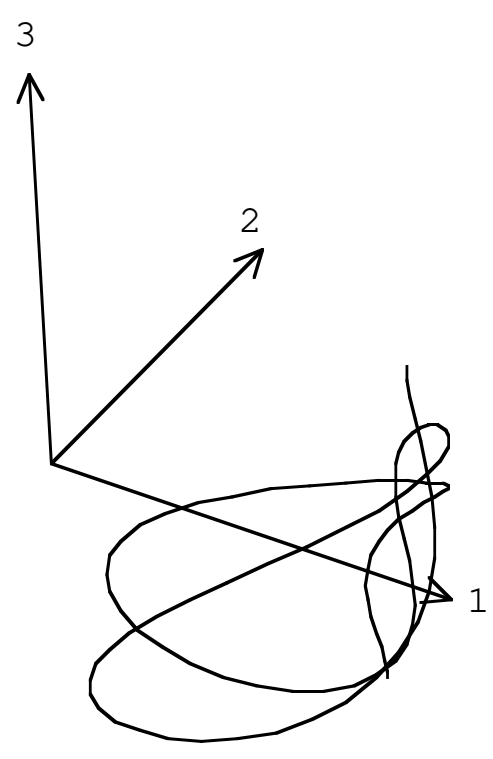

(b)

Figure 8 


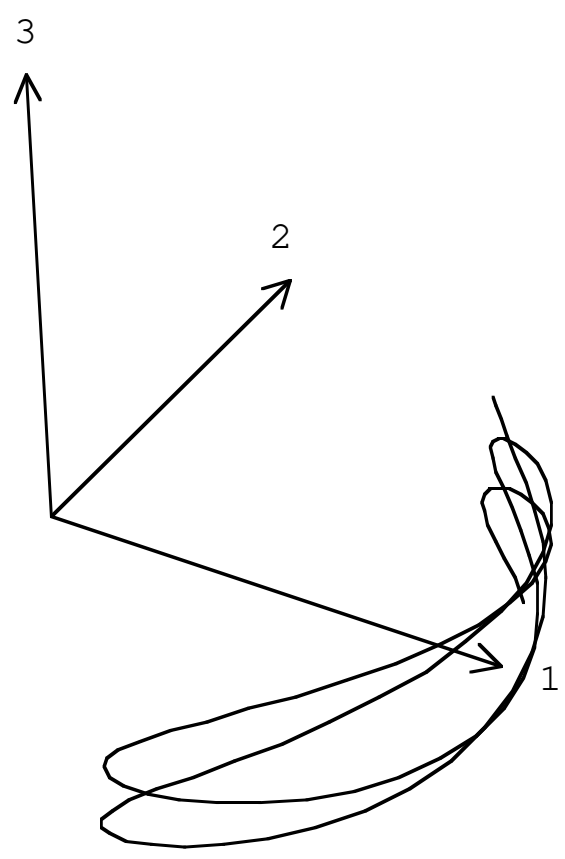

(a)

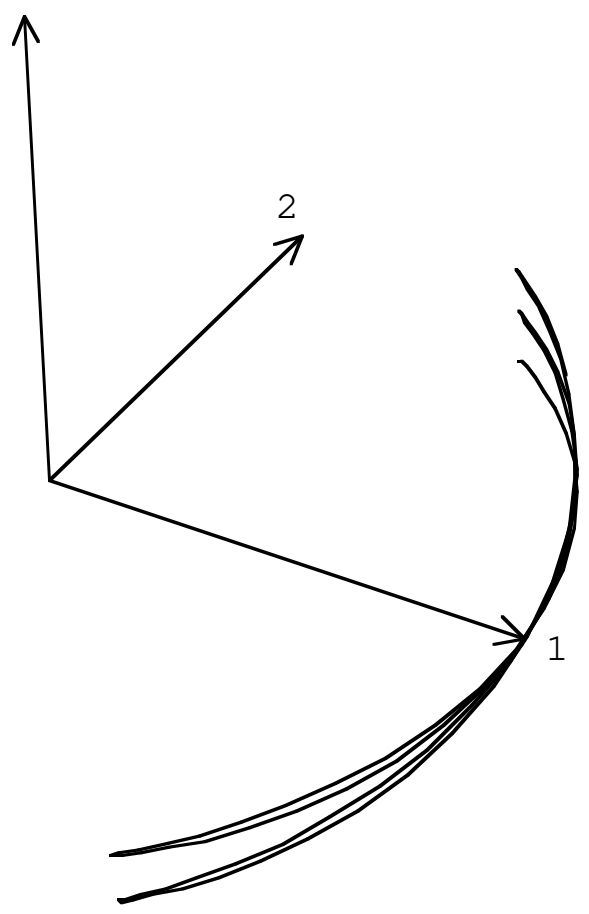

(b)

Figure 9 


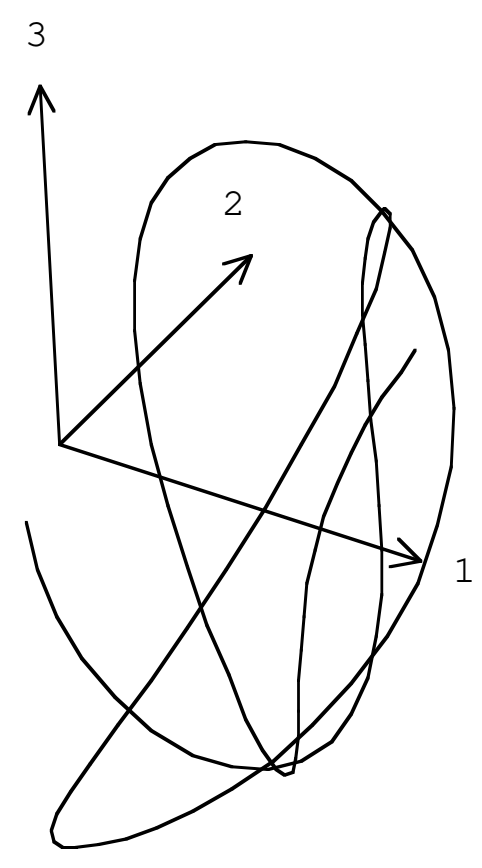

(a)

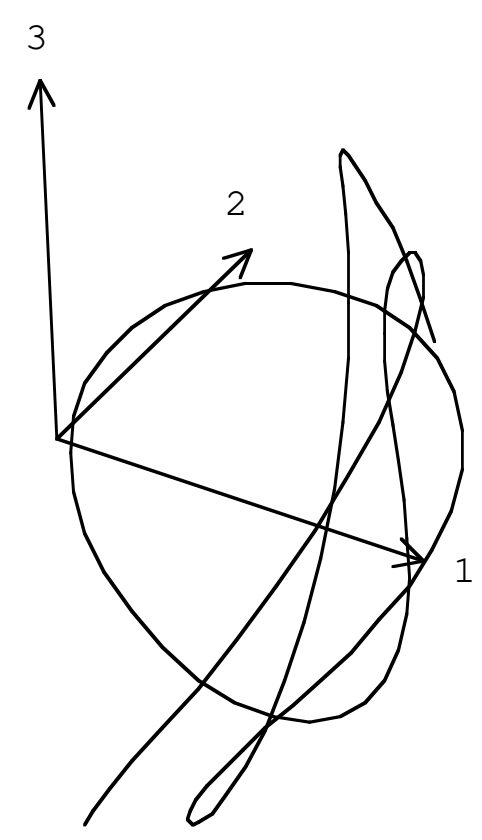

(b)

Figure 10 


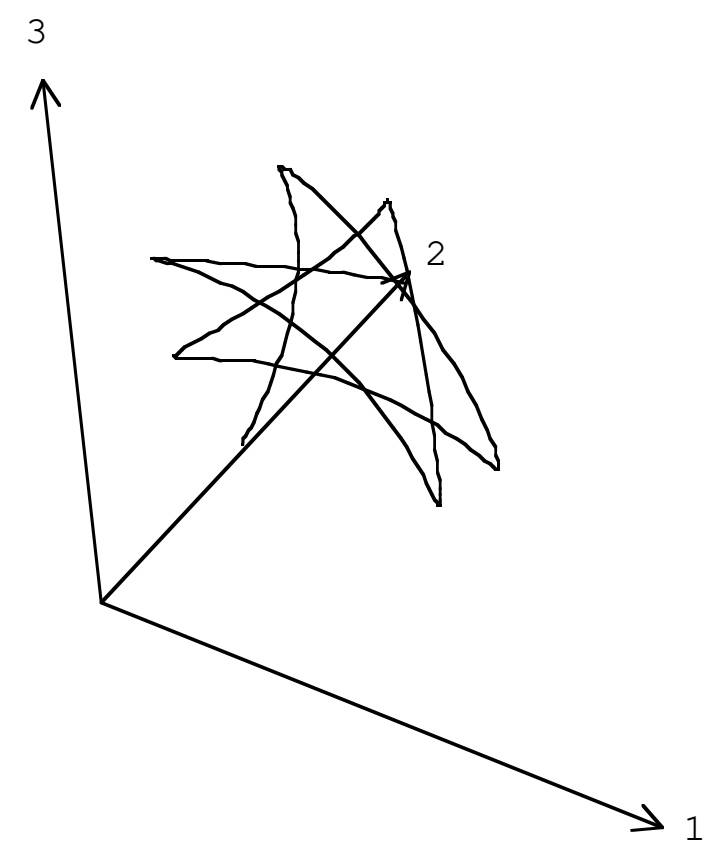

Figure 11 